\title{
Quantification of geometric errors made simple: application to main-group molecular structures
}

\author{
Stefan Vuckovic ${ }^{*, \dagger, \ddagger}$ \\ $\dagger$ Institute for Microelectronics and Microsystems (CNR-IMM), Via Monteroni, Campus \\ Unisalento, 73100 Lecce, Italy \\ $\ddagger$ Department of Chemistry 85 Pharmaceutical Sciences and Amsterdam Institute of \\ Molecular and Life Sciences (AIMMS), Faculty of Science, Vrije Universiteit, De Boelelaan \\ 1083, 1081HV Amsterdam, The Netherlands \\ E-mail: s.vuckovic@vu.nl
}

\section{Abstract}

Nearly all electronic structure simulations begin with obtaining approximate geometries, making a systematic quantification of errors in approximate molecular structures of key importance. Recently, the geometric energy offset (GEO) framework based on a single and natural measure for quantifying and analysing these errors has been proposed [J. Phys. Chem. Lett. 2020, 11, 99579964]. An accurate and way less costly approximation to GEO is utilized here to readily quantify errors in main-group structures and analyze them in a chemically intuitive way. The use of semiexperimental geometries as a reference further simplifies the analysis. The analysis reveals new insights into the geometric performance of methods, their rankings, as well as patterns across different classes of methods and basis sets that arise from the analysis.

\section{Introduction and back- ground}

The use of electronic structure calculations to rational, guide and support experiments has become a routine in different branches of science. Such practical calculations, be they based on wave-function or density functional theory (DFT) approximations, conflate errors in both approximate molecular geometries and total energies. Thus, a proper analysis of the performance of electronic structure methods requires decoupling these two distinct sources of errors and their separate analysis. The energetic performance of electronic structure methods is often drastically different from their performance for molecular geometries. Methods with comparable energetic performance, for e.g., binding energies of noncovalent systems, can have strikingly different geometric performance for the same systems. 1 Other examples include Hartree-Fock (HF) and the local density approximation (LDA), which give reasonable structures despite their poor energetic performance. Nearly all electronic structure simulations (and beyond) begin with obtaining approximate geometries, making a proper and extensive quantification of geometric errors of key importance in computational chemistry.

By using the standard tools for comparing structures, it is not easy to tell which of approximate geometries is better, as it requires comparing errors in $3 N-6$ degrees of freedom. What typically happens is that some geometric parameters are more accurate in one method, some are better in another (see, e.g., Refs. 1-3). The geometric performance of electronic structure methods is commonly assessed by comparing averages of errors in these parameters (e.g., bond lengths, angles, distances from a chosen 
point in a molecule such as the center of mass, etc. $)^{3-9}$ But, when such metrics are used, the rankings of approximations strongly depends on a chosen metric as illustrated in detail in Ref. 1. By comparing errors in geometric parameters, it is not trivial to tell which of the approximations yields an overall better geometry even for systems with two degrees of freedom (e.g., wa$\operatorname{ter}^{(1)}$, as one approximation can beat the other for the first degree of freedom, but not for the second. Other ambiguities that affect the rankings based on this approach also arise, such as: whether one should average errors over all bond lengths/angles or only unique ones.

In a recent work, Vuckovic and Burke (VB) introduced a framework for quantifying and analysing errors in approximate geometries based on the concept of geometry energy offset $(G E O)$. GEO provides a remarkably simple and intuitive approach for quantifying errors in molecular geometries, which by-passes the need to compare the errors in individual geometric parameters. For a given approximate geometry, $G E O$ is simply defined as the difference in exact energies at the approximate and exact geometries:

$$
G E O=E(\tilde{\mathbf{G}})-E\left(\mathbf{G}_{0}\right),
$$

where $E(\mathbf{G})$ is the ground-state energy at geometry $\mathbf{G}, \mathbf{G}_{0}$ the exact geometry and $\tilde{\mathbf{G}}$ an approximate geometry. The theoretically exact geometry is defined here as the exact minimum of the exact ground-state potential energy surface, and the Born-Oppenheimer approximation is assumed throughout this work. Defined this way, GEO represents an energetic distance between the exact and approximate geometries, and thus provides a single number measure for the quality of geometries. At minima, GEO vanishes only if $\tilde{\mathbf{G}}=\mathbf{G}_{0}$, and otherwise it is always a positive number given in energy units. Thereby, GEO provides an unambiguous measure for the quality of approximate geometries (the higher the $G E O$ value, the worse the geometry). As such, it circumvents a need for comparing errors in possibly dozens of bond lengths and angles to rank approximations. These features make $G E O$ an ideal quantity for assess- ing the quality of geometries of any molecule and any method so long as the reference geometries are available. Furthermore, GEO can also tell us what fraction of the total error is due to geometry, and what due to total energy. In Appendix A, we show the decomposition of the total error of an electronic structure calculation into geometric and non-geometric part. This decomposition has shown that geometric errors are typically small (but not negligible) part of errors for e.g., atomization energies, but can account for most of the error for weak interactions. ${ }^{1}$ It also enabled identification of specific situations where better results are obtained by using less accurate structures due to error cancellations between the geometric and nongeometric part of the total error. ${ }^{1}$

Computing GEO by Eq. 1 requires access to the exact geometries and exact energies at approximate geometries. For single-reference systems, $\operatorname{CCSD}(\mathrm{T})$ with large enough basis set provide accuracy that rivals experimental geometries, 10 so we can consider them 'exact'. VB used $\operatorname{CCSD}(\mathrm{T}) /$ aug'-cc-pV5Z $^{11112}$ as a reference to calculate $G E O$ for a set of small molecules that had been earlier optimized by Karton and co-workers. $\stackrel{11}{ }$ For somewhat larger systems (e.g., aromatics containing two benzene rings), running $\mathrm{CCSD}(\mathrm{T})$ geometry optimizations with a large basis set becomes too expensive. For this reason, VB had to rely on B2PLYP as a proxy reference, $\frac{13}{13}$ given its nearly CCSD $(\mathrm{T})$ performance for small molecular structures. In a more recent work, Bakowies and von Lilienfeld extended this analysis to a larger set of small molecules, and even built empirical corrections for the part of atomization energies error that is due to approximate geometries. 14

Here an important question arises: When accurate reference geometries are available, can the use of expensive single point calculations at approximate geometries needed to calculate GEO by Eq. 1 be by-passed? Such calculations make the benchmarking of geometries more expensive, and thus pose the restrictions regarding the molecular size. The answer to this question is yes, as assessing geometric performance of approximations by calculating $G E O$ values 

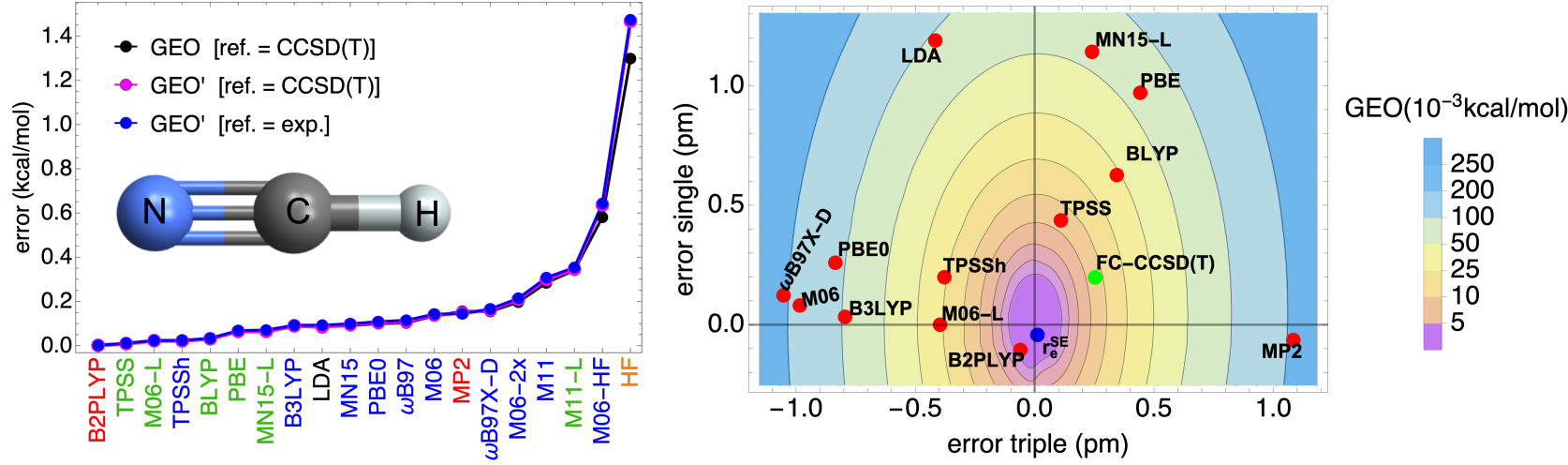

Figure 1: Left panel: $G E O$ with $\operatorname{CCSD}(\mathrm{T})$ as reference (black), and $G E O^{\prime}$ with both $\mathrm{CCSD}(\mathrm{T})$ (magenta) and semiexperimental structures ${ }^{15}$ (blue) used as a reference for the HCN molecule. allelectron $\operatorname{CCSD}(\mathrm{T})$ has been used as a reference in all calculations in tandem with aug-cc-pCV5Z for $\mathrm{C}$ and $\mathrm{N}$ atoms, and cc-pV5Z for the $\mathrm{H}$ atom. For all approximations (DFT and MP2), aug-ccpVQZ basis set has been employed. Right panel: GEO contours as a function of errors in the triple and single bond for the same molecule and the positions of selected approximation with $\mathrm{CCSD}(\mathrm{T})$ as a reference. The position of semiexperimental results is denoted by a blue circle.

can be greatly simplified by introducing its approximation: 1

$$
G E O^{\prime}=\tilde{E}\left(\mathbf{G}_{0}\right)-\tilde{E}(\tilde{\mathbf{G}})
$$

For covalent systems, GEO is excellently approximated by $G E O^{\prime}$ and provides essentially the same information (e.g., rankings of approximations, the decomposition of the error into contributions from the errors in different structural parameters, etc. $)^{1}$ At the same time, computing $G E O^{\prime}$ is way less costly as it does not require running a single-point calculation with a high-level method, such as $\operatorname{CCSD}(\mathrm{T})$. Furthermore, if a reference geometry is derived from an experiment, we no longer need $\operatorname{CCSD}(\mathrm{T})$ at all, as for $G E O^{\prime}$ we only need approximate energies at both reference and approximate geometries (Eq. 2). In practice this can be done by using accurate geometries as a starting point for a geometry optimization with an approximate method. After the convergence, one can easily calculate $G E O^{\prime}$ from the differences in approximate energies from the first and last iteration of the optimization procedure.

In the present paper, the advantages of $G E O^{\prime}$ over $G E O$ are used for a systematic analysis of geometric performance of approximations for main-group structures. By using $G E O^{\prime}$ in place of $G E O$ in tandem with accurate semiexperimental geometries, $15-17$ the analysis does not rely on the expensive CCSD $(\mathrm{T})$ calculations nor on a proxy reference as it was the case in the previous study. 1 While the previous study focused on the GEO analysis at a fixed basis set, here we observe how the changes in a basis set affect the geometric performance of approximations. From this analysis, we find that some of the worst performers at a large basis sets are one of the top performers at a smaller basis due to error cancellations. We dedicate special attention to the geometric performance of different classes of DFT methods, and analyze how it varies with the amount of exact exchange. A harmonic approximation to $G E O^{\prime}$ enables us to directly link and partition $G E O^{\prime}$ into contributions from errors in specific geometric parameters. This analysis reveals different patterns for different classes of DFT approximations and tells us how these patterns change with a basis set size.

The paper is organized as follows. The stage is set in Sec. 2, where the the differences between $G E O$ and $G E O^{\prime}$ are examined by using the $\mathrm{HCN}$ molecule as an example. In the same section, the set of accurate semiexperi- 
mental geometries is validated for the purpose of calculating $G E O^{\prime}$ in the present work. The main results are in the next two sections, with Section 3 focusing on the quantification of geometric errors and trends across approximations, and Section 4 focusing on the $G E O^{\prime}$ analysis (a breakdown of $G E O^{\prime}$ into contributions from different geometric parameters). The last section is devoted to conclusions and discussion on where the $G E O$ approach should prove powerful in the future.

\section{Setting the stage}

\subsection{The simple HCN example}

To compare $G E O$ with $G E O^{\prime}$ and get a feeling how the errors in geometric parameters translate into $G E O$, we use the HCN molecule. The $\operatorname{CCSD}(\mathrm{T})$ (full) method is taken as a reference with the aug'-cc-pCV5Z basis set [cc-pCV5Z for the hydrogen atom and aug-cc-pCV5Z for the other two atoms ${ }^{18}$, and (full) indicates that all electrons are included in the correlation treatment.

The right panel uses $G E O$ values to rank various approximation for the $\mathrm{HCN}$ structure (black line). Both sets of $G E O^{\prime}$ values, the one that uses values the $\operatorname{CCSD}(\mathrm{T})$ structure (magenta) and the one that uses semiexperimental (SE) structure (blue) are virtually indistinguishable from $G E O^{\prime}$. This is the case even though $G E O$ costs way more to compute than $G E O^{\prime}$. The use of $G E O^{\prime}$ in place of $G E O$ enables us to bypass the use of expensive $\operatorname{CCSD}(\mathrm{T})$ energies to perform the $G E O$ analysis. For $G E O^{\prime}$ we also need reference $\mathbf{G}_{0}$ (Eq. 2), but the use of SE geometries enables us to completely by-pass input from $\operatorname{CCSD}(\mathrm{T})$ and extend the $G E O^{\prime}$ analysis to molecules whose structures cannot be obtained by $\operatorname{CCSD}(\mathrm{T})$ (full) within a sufficiently large basis set. In general, $G E O^{\prime}$ is energetically very close to $G E O$ even for inaccurate methods, such as $\mathrm{HF}^{1}, 1$ as the curvature of $\tilde{E}(\mathbf{G})$ at the minimum is reasonable even for HF (compare Eqs. 6 and 7).

To see how the errors in geometric parameters of HCN translate to GEO, the $G E O$ contours are plotted in the right panel of Figure 1. This is done by calculating the $\operatorname{CCSD}(\mathrm{T})$ (full) potential energy surface around the equilibrium geometry. The molecule is linear and all tested approximations get that right. Thus, in the contour plot we show only errors in the two geometric parameters: the $\mathrm{C} \equiv \mathrm{N}$ (x-axis) and $\mathrm{C}-\mathrm{H}$ (y-axis) bond lengths. The poorest performers are HF, M11-L, M06-HF, whose errors are beyond the shown range. First we note that the SE structure (blue dot) is in an excellent agreement with the reference. The B2PLYP geometry is in a very good agreement with both SE and CCSD(T) structures, and has negligibly small $G E O$ value.

It is commonplace to run $\operatorname{CCSD}(\mathrm{T})$ calculations with the frozen core (FC) approximation, and if this approximation is turned on for CCSD(T)/aug'-cc-pCV5Z, the structure is still accurate (green dot), but it is off the center. This confirms that apart from a large basis set one also needs all-electron $\operatorname{CCSD}(\mathrm{T})$ to rival the accuracy of the SE approach. $\frac{10}{10}$ This also demonstrates the advantage of using the SE structures as running $\operatorname{CCSD}(\mathrm{T})$ (full) with a large basis can only be done for very small molecules. When it comes to the DFT approximations in the $G E O$ contour plot, one can observe their clustering, as previously observed for water. 1 Here, the hybrid functionals are clustered together in the first quadrant (too short triple bond and accurate single bond), and metaGGA/GGAs are in the second quadrant (predicting too long bonds with larger errors for the single bond). M06-L ${ }^{19}$ is an exception, but as we shall see later, its performance for geometric structures is more in-line with hybrids than with metaGGAs.

\subsection{A dataset of semiexperimen- tal geometries used for $G E O^{\prime}$ evaluations}

In the remainder of this work, reference geometries ('exact' $\mathbf{G}_{0}$ ones entering Eq. 2) are taken from the B2se dataset of Barone and coworkers. 15 This dataset contains accurate equi- 
librium structures of 68 molecules that have been obtained from the SE approach. Within the SE approach, equilibrium structures are derived from experimental ground-state rotational constants in which vibrational contributions computed by a suitable quantum mechanical (QM) methods are subtracted.15] 17 Thereby, the SE structures match our definition of $\mathbf{G}_{0}$, as they correspond to the minima on the Born-Oppenheimer potential energy surface. In terms of applicability and accuracy, the SE approach offers a range of advantages for accurate structure determinations over only experimental or only theoretical approaches (see, e.g., Refs. 15,16). The B2se structures employed here are shown in the SI (see Fig. S1), and the phenyl radical, as the only open-shell B2se species is excluded and later separately analysed in Section 4.4 .

Barone and co-workers have built the B2se set by using B2PLYP/cc-pVTZ to compute vibrational corrections. 15 This level of theory gives nearly identical structures to those computed from CCSD $(\mathrm{T})$ vibrational corrections. ${ }^{15}$ For a subset of the B2se structures, vibrational correction have been computed at the $\operatorname{CCSD}(\mathrm{T})$ with at least a triple- $\zeta$ basis set and these are contained in the CCse set. 10 To validate the use of the B2se structures as a reference, we calculate $G E O^{\prime}$ values for a range of approximate structures by using both B2se and CCse geometries as a reference. This is done for a subset of the B2se set for which the CCse geometries are available. The two sets of $G E O^{\prime}$ values (B2se vs. CCse as a reference) are essentially the same (typically within $0.005 \mathrm{kcal} / \mathrm{mol}$ ), as shown in Fig. S2 of the SI. This confirms the suitability of the B2se structures as reference geometries for performing the $G E O$ analysis in the present work.

\section{Quantification of geo- metric errors by $G E O^{\prime}$ and trends across ap- proximations}

\subsection{The $G E O^{\prime}$ rankings of ap- proximations and basis set trends}

In Figure 2, the rankings of various approximations (DFT methods, HF and MP2) based on the average $G E O^{\prime}$ values for the B2se dataset are shown. The aug-cc-pVnZ (AVnZ) basis set is used, with $n=\mathrm{Q}$ in the left, $n=\mathrm{T}$ in the middle, and $n=\mathrm{D}$ in the right panel. The approximations are ranked by their mean $G E O^{\prime}$ values at the AVQZ basis set, so one can see how the rankings are affected as the basis set size decreases.

First we focus on the AVQZ panel of Figure 2. B2PLYP (a double hybrid) $\frac{13}{13}$ is the winner and HF has expectedly the worst performance. Hybrids typically perform better than semilocal DFT functionals. An exception is M06-L that has an excellent performance, which is more in line with hybrid functionals than with other tested semilocal functionals. On the other hand, M06-HF ${ }^{20}$ performs much worse than other hybrids. LDA's $G E O^{\prime}$ is comparable to that of $\mathrm{PBE}^{21}$ and even better than BLYP.22123 This makes the geometric performance of LDA remarkable relative to its poor energetic performance (for, e.g., atomization energies). ${ }^{2124} \mathrm{M} 11-\mathrm{L}, \stackrel{25}{25}$ as observed earlier by Jacquemin and co-workers, 26 displays very bad performance for molecular geometries and it is here just slightly better than HF.

Moving from the AVQZ to AVTZ panel (middle), the rankings are mostly preserved with some exceptions. B2PLYP is still the winner, $\mathrm{HF}$ is still the worst, but MP2 now loses to most of the hybrids. The gap between hybrids and semilocal functionals is now bigger. The exception is M06-L again, which now beats all tested hybrids.

Much more abrupt changes in the rankings are seen as one goes from AVTZ to AVDZ (the right panel): M06-2x $\mathrm{x}^{27}$ is now the best, MP2 is behind LDA and beats only PBE and BLYP, and B2PLYP is now worse than all of the hybrids. Surprisingly, the performance of HF strikingly improved, and it is now in front of all semilocal functionals except for M06-L. Interestingly, GEO' of HF/AVDZ is about a half 

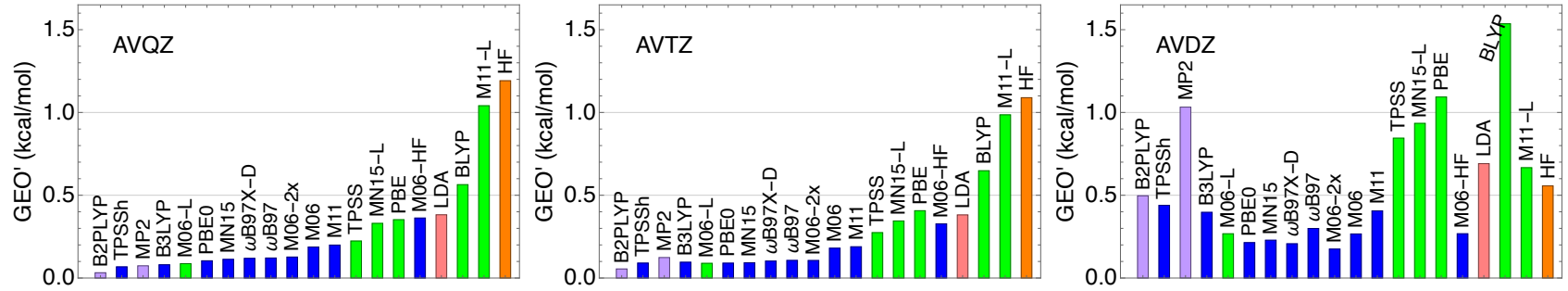

Figure 2: Mean $G E O^{\prime}$ values for approximations for the B2se dataset within different basis sets: AVQZ (left), AVTZ (center), AVDZ (right). Purple bars denote B2PLYP and MP2, blue bars denote hybrids, green bars denote meta-GGAs and GGAs, pink denotes LDA and orange color is used for the HF method. The approximations in all three panels are ranked by their AVQZ GEO' values (left panel).

of that of HF/AVQZ and a half of MP2/AVDZ. This clearly suggest that this surprisingly good geometric performance of $\mathrm{HF} / \mathrm{AVDZ}$ is due to the error cancellation between the absence of correlation and a large basis set incompleteness error.

To shed more light on the different $G E O^{\prime}$ trends with the basis set size, in Figure 3 we show the $G E O^{\prime}$ boxplots for MP2, B3LYP, M06-2x and HF at the three basis sets (for the same plot for other methods, see Figure S4). Each of these approximations behaves differently. MP2 is a disaster at AVDZ and then it dramatically improves at AVTZ. A significant improvement is also observed as one goes from AVTZ to AVQZ. In the case of B3LYP, the spread is also larger as the basis set decreases but expectedly much less drastic than it is the case with MP2. M06-2X is interesting because its $G E O^{\prime}$ trends change very little with the basis set size. This is also the case with other Minnesota functionals except for MN15$\mathrm{L}^{28}$ (see Figure $\mathrm{S} 4$, where we show the same boxplots for all tested approximations). HF displays a unique trend here: the spread becomes smaller as the basis set size decreases. This behaviour has also an impact on the $G E O^{\prime}$ performance on hybrids, and in Section 3.4, we study in more detail how the amount of exact exchange affects the geometric performance of hybrids.

Since the inclusion of diffuse functions in a basis set is often too expensive for the geometry optimizations, we also test here what happens with the $G E O^{\prime}$ performance of approximations as one goes from the $\mathrm{AVnZ}$ to $\mathrm{V} n \mathrm{Z}$ basis set. The results are shown in Figure 4, where in each of the panels the ranking is kept of the respective AVnZ basis set. From this figure, we can see that the omission of the diffuse functions does not much affect the rankings of approximations, but it typically increases average $G E O^{\prime}$. Expectedly, it does more so as one goes from AVQZ to AVDZ. In the case of hybrids, the AVnZ to $\mathrm{V} n \mathrm{Z}$ change worsens the average $G E O^{\prime}$ by no more than $5 \%$ when $n=\mathrm{Q}$ or $n=\mathrm{T}$, but it can worsen by $\sim 40 \%$ when $n=\mathrm{D}$.

\subsection{How does dispersion correc- tions affect DFT $G E O^{\prime}$ values for the B2se set?}

In Figures 2, 4, the DFT approximations are employed without empirical dispersion corrections as these have a little effect on the geometric performance given the relatively small size of the B2se molecules. To see the effect of the dispersion correction for the B2se geometries, in Figure 5 we show a parity plot comparing $G E O^{\prime}$ of TPSS (a metaGGA) enhanced by D3(BJ) against that of bare TPSS. D3(BJ) denotes Grimme's dispersion correction $^{29}$ with the dumping function of Becke and Johnson. 30131 The GEO' values for BLYP and $\mathrm{TPSSh}^{32}$ are also shown for comparison. We can see from the plot that the addition of D3(BJ) has almost no effect on the $G E O^{\prime}$ values of TPSS. On the contrary, the effect of exact exchange addition is way more profound as the $G E O^{\prime}$ values of TPSSh, which contains $10 \%$ of 

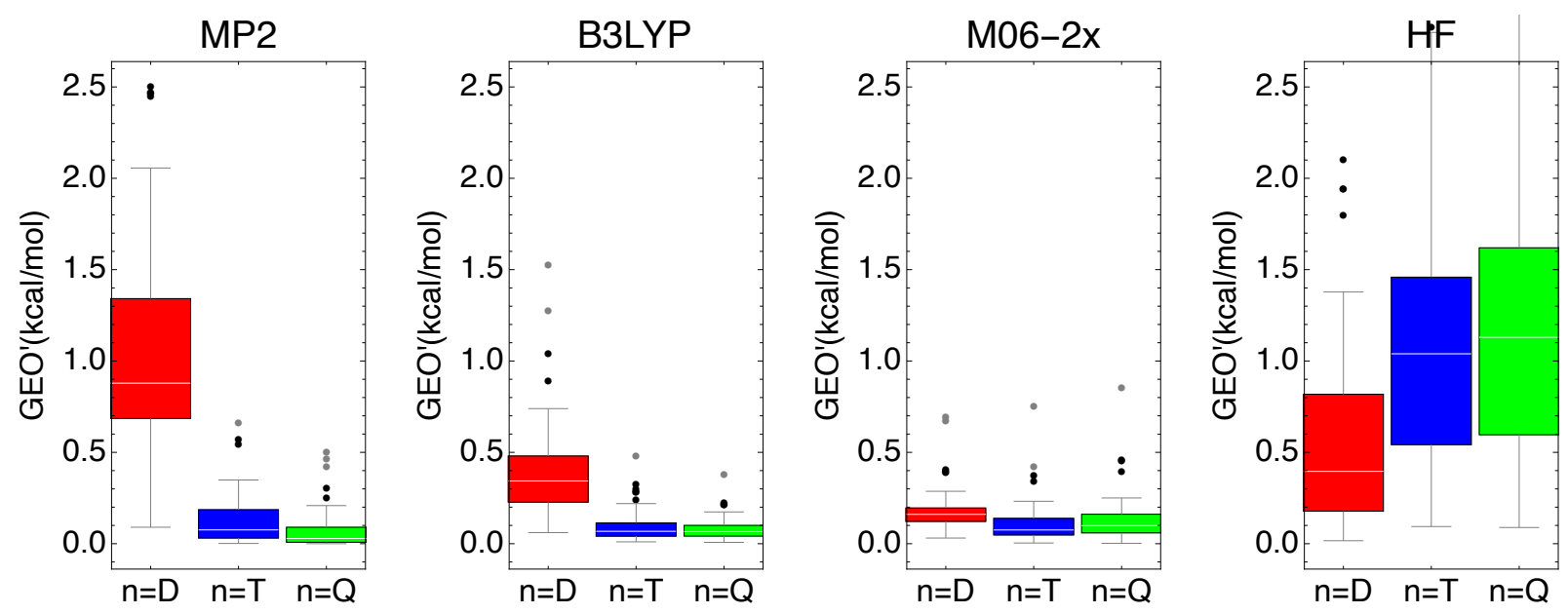

Figure 3: Boxplots of the B2se dataset showing $G E O^{\prime}$ for selected approximations within the $\mathrm{AV} n \mathrm{Z}$ basis set, with $\mathrm{n}=\{\mathrm{Q}, \mathrm{D}, \mathrm{T}\}$. The central $50 \%$ of the $G E O^{\prime}$ datapoints are bounded by boxes, whiskers bound all data points except for the outliers which are beyond 1.5 times the interquartile range of the box edges and are represented by dots. White lines mark median values. For boxplots of other approximations and $\gamma^{\prime}$ version of boxplots, see Figures S4, S6 in the SI.
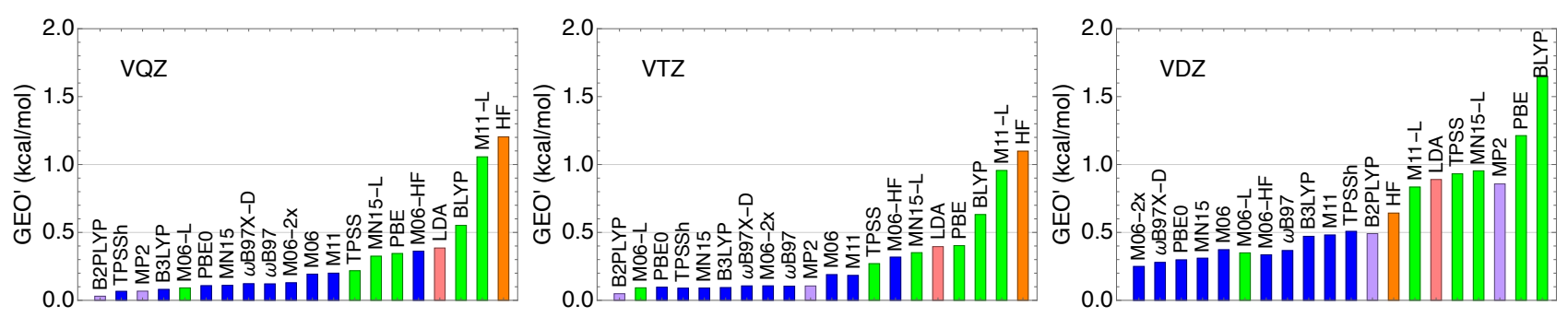

Figure 4: Same as Figure 2, but within the VnZ basis set (no diffuse functions). The approximations in all three panels are sorted by their respective $\mathrm{AVnZ} G E O^{\prime}$ rankings so one can see how the exclusion of the diffuse functions from the basis affects the rankings of approximations. For the same plots within the VnZ basis set, see Figure S3

exact exchange, are about one-third of those of TPSS. Of course, for larger molecules and weak interactions, we expect that dispersion corrections would have a larger effect on DFT GEO' values. 111433

\subsection{GEO' rankings of approxi- mations on the absolute $G E O$ scale}

As discussed in Ref. 1, GEO and $G E O^{\prime}$ values increase with the molecular size. That is why the $G E O$ absolute scale has been introduced as the $G E O^{\prime}$ values relative to it do not increase as molecules grow. To introduce the absolute $G E O$ scale, we first define $\Delta \mathbf{G}$ as the error in approximate geometry: $\Delta \mathbf{G}=\tilde{\mathbf{G}}-\mathbf{G}_{0}$. Setting $\Delta \mathbf{G}=\gamma \mathbf{G}_{0}$, produces to second order in $\gamma$ :

$$
G E O_{\gamma}^{\prime}=\gamma^{2} \tilde{D} / 2
$$

where,

$$
\tilde{D}=\tilde{\mathbf{G}}^{\top} \tilde{\mathbf{H}} \tilde{\mathbf{G}},
$$

where $\tilde{\mathbf{H}}$ is the Hessian of $\tilde{E}(\mathbf{G})$ at the $\tilde{\mathbf{G}}$ minimum. $\tilde{D}$ is here the absolute $G E O$ scale, which by $\mathrm{Eq} 3$ gives us $G E O^{\prime}$ value when the exact geometry is compressed (or expanded) by $\gamma$. If $\gamma=1 \%, G E O_{\gamma}^{\prime}$ values for the B2se molecular are in a narrow interval in between 0.1 to 1.2 $\mathrm{kcal} / \mathrm{mol}$ with the average $G E O_{\gamma}^{\prime}$ being about $0.5 \mathrm{kcal} / \mathrm{mol}$. The $\tilde{D}$ value varies little across approximations. For example, B3LYP $\tilde{D}$ val- 


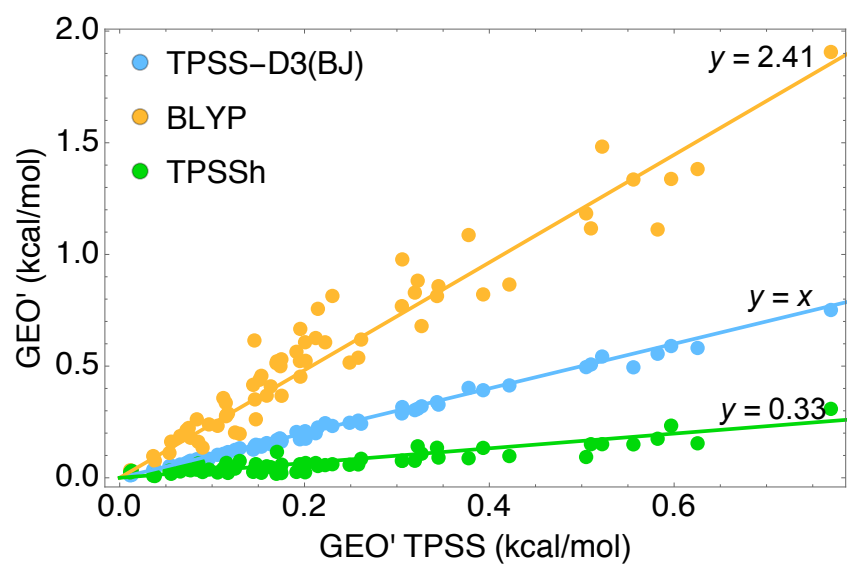

Figure 5: GEO' values of TPSS-D3(BJ), BLYP, and TPSSh vs. GEO' values of TPSS for the B2se molecules. The coefficients in the linear fits are obtained from the ratios between MAEs of the three methods and MAE of TPSS. The AVQZ basis set is used in all calculations.

ues are within $1 \%$ of $\operatorname{CCSD}(\mathrm{T})$ ones and even HF gives reasonable values. ${ }^{1}$ Thus, the $\tilde{D}$ values are always computed here at the B3LYP/AVTZ level, and are reported for the B2se molecules in Figure S1. As said, the main idea of $\tilde{D}$ is to give a scale on which $G E O^{\prime}$ does grow with a molecular size. Thus, we define:

$$
\gamma^{\prime}=\sqrt{\frac{2 G E O^{\prime}}{\tilde{D}}},
$$

by equating $G E O^{\prime}$ of Eq. 2 with $G E O_{\gamma}^{\prime}$ of Eq. 3. In this way, $G E O^{\prime}$ from a given approximation would also be obtained if $\gamma^{\prime}$ were a relative error in all bond lengths and there were no errors in angles.

If $\gamma^{\prime}$ is now used in place of $G E O^{\prime}$ to rank the approximations, the rankings are preserved with few exceptions when the differences in numbers are marginal. This is illustrated in Figure 6, where we repeat Figure 2, but with bars denoting mean $\gamma^{\prime}$ values of approximations, which range from $\sim 0.2 \%$ to $\sim 1.7 \%$. If one wants to estimate $G E O^{\prime}$ values for a molecule that is larger than those of B2se, it can be done from: $G E O^{\prime} \sim \gamma^{\prime 2} \tilde{D} / 2$, by using the average $\gamma^{\prime}$ values reported in Figure 6 and $K$ of that specific molecule.

\subsection{How does geometric perfor- mance of DFT approxima- tions vary with the amount of exact exchange?}

In this section we focus on how the geometric performance of DFT methods varies with their amount of exact exchange. For this purpose, we employ the $\alpha$-PBE hybrid which is built from the PBE functional by replacing the $\alpha$ amount of PBE exchange with the same amount of exact exchange. At $\alpha=0.25, \alpha$-PBE becomes PBE0. 3435

The mean B2se's GEO' values for $\alpha$ $\mathrm{PBE} / \mathrm{AV} n \mathrm{Z}(n=\{\mathrm{Q}, \mathrm{D}, \mathrm{T}\})$ are shown in the top panel of Figure 7 (note the $\log$-scale in the $\mathrm{y}$-axis). In terms of the shape of the curves, their ranges, and the position of the minima, AVTZ curve is similar to AVQZ, but very different from the AVDZ. Up to $\alpha \sim 0.3$, the mean $G E O^{\prime}$ is lower with AVTZ than with AVDZ. At larger $\alpha$ values ( $\alpha$ greater than $\sim 0.3$ ), AVDZ becomes more accurate than AVTZ. This behaviour of $\alpha-\mathrm{PBE}$ can be traced back to the geometric performance of HF, given that: (i) $\alpha$-PBE becomes more similar to HF as $\alpha$ approaches 1, (ii) HF's $G E O^{\prime}$ is much lower with AVDZ than AVTZ. The minima of the AVQZ and AVTZ curves are at $\alpha \sim 0.2$, whereas that of the AVDZ curve is expectedly shifted towards larger $\alpha(\alpha=0.36)$. Around these values we can also find most of the optimal $\alpha$ values for $G E O^{\prime}$ of the individual B2se molecules (see Figure S9]. For example, more than $70 \%$ of the optimal $\alpha$ values within the AVTZ basis set for the individual B2se structure lie in between 0.17 and 0.24 . If we look at the mean $\gamma^{\prime}$ (in place of mean $G E O^{\prime}$ ) as a function of $\alpha$, the minima are still at about the same $\alpha$ values (see the inset in the top panel of Figure 7).

The bottom panel of Figure 7 zooms in on the region about the minima of the AVDZ and AVTZ curves, and there we add datapoints of the mean $G E O^{\prime}$ values of functionals containing different amounts of the exact exchange. From there we can see that the performance of TPSSh and B3LYP is nearly the same as that of respective $\alpha$-PBE. On the other hand, Minnesota 

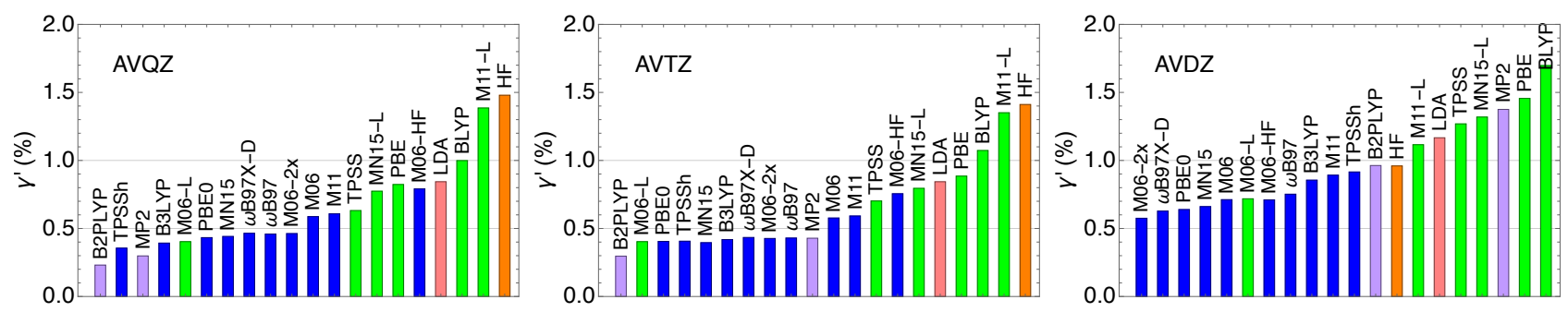

Figure 6: Mean $\gamma^{\prime}$ values (Eq. 5) of selected approximations within the three basis sets. The approximations in all three panels are sorted by their respective AVnZ GEO' rankings.
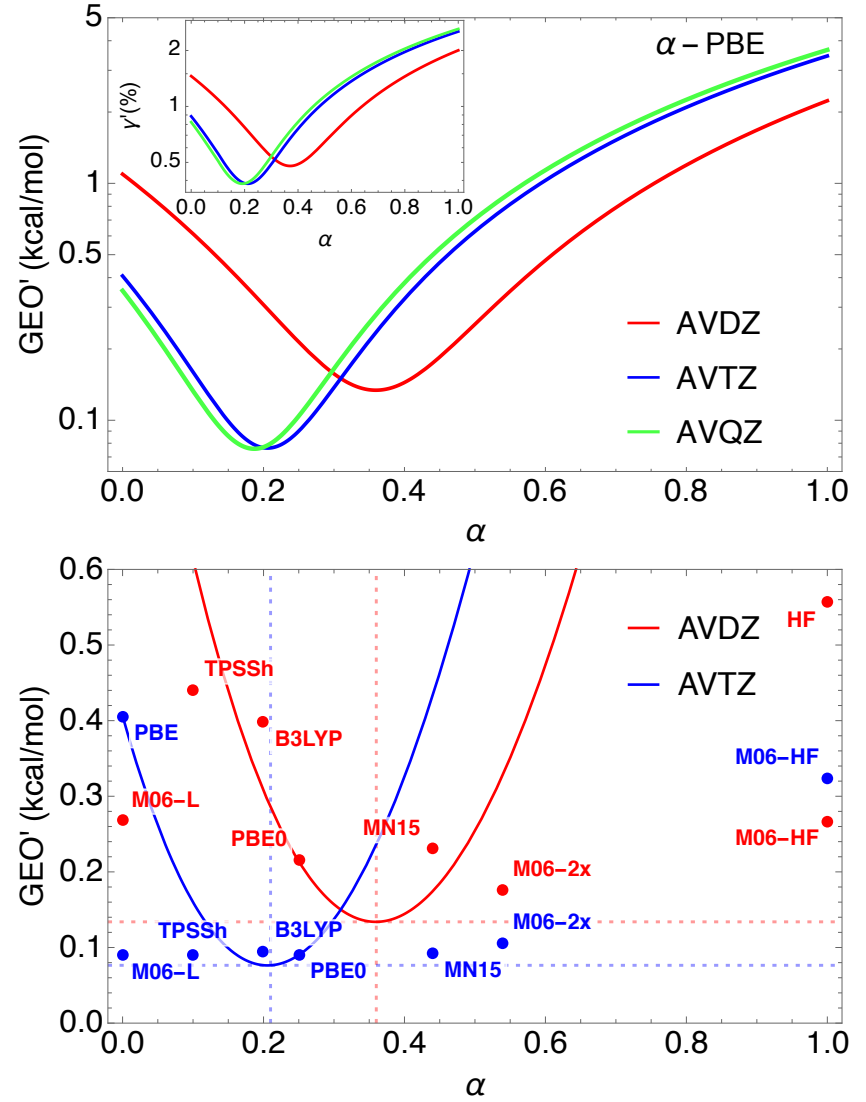

Figure 7: Top panel: the mean $G E O^{\prime}$ values of the $\alpha-\mathrm{PBE} / \mathrm{AV} n \mathrm{Z}$ as a function of $\alpha$ with $n=\{\mathrm{Q}, \mathrm{D}, \mathrm{T}\}$, where $\alpha$ denotes the amount of exact exchange. Note the log-scale on the yaxes. The inset in the top panel shows the same curves but with the mean $\gamma^{\prime}$ in place of the mean $G E O^{\prime}$ values. Bottom panel: AVTZ and AVDZ from the top panel zoomed around their minima with the $G E O^{\prime}$ datapoints for other functionals with different amounts of exact exchange. For $\gamma^{\prime}$ version of the bottom panel, see Fig. ST7.

functionals are typically better than the respective $\alpha$-PBE functional (note that Minnesota functionals are designed so that their exchange and correlation parts fit each other). Only MN15/AVDZ gets beaten by $\alpha$-PBE within the same amount of exact exchange and basis set, given the very good performance of $\alpha$ $\mathrm{PBE} / \mathrm{AVDZ}$ in the region around that $\alpha$ value. Finally, $\alpha$-PBE at the optimal $\alpha=0.36$ value outperforms all functionals with AVDZ. When VDZ is used in place of AVDZ, the curve has nearly the same shape but is shifted upwards by $\sim 0.1 \mathrm{kcal} / \mathrm{mol}$ (see Figure S8).

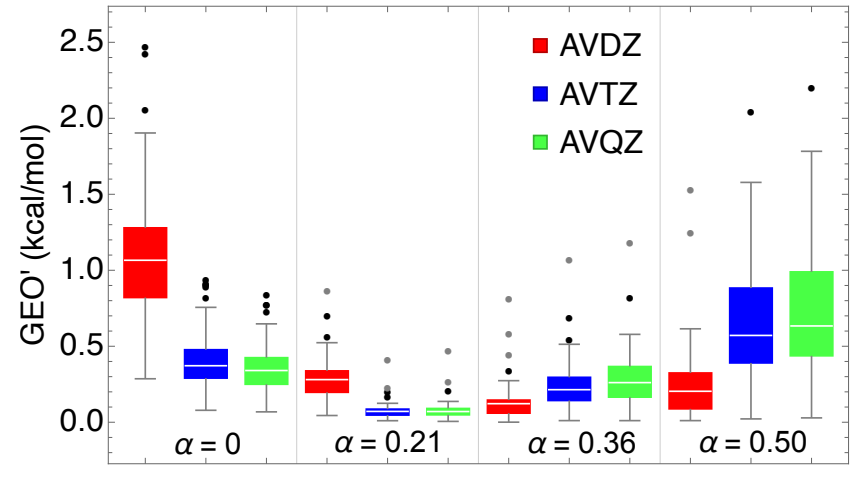

Figure 8: Boxplots same as those in Fig. 3, but for $\alpha$-PBE at the four $\alpha$ values.

In Figure 8, we also show the $G E O^{\prime}$ boxplots of $\alpha$-PBE for the B2se at the following $\alpha$ values: $0,0.21$ (the minimum of the AVTZ curve), 0.36 (the minimum of the AVDZ curve), 0.5. From these boxplots, we can see that at the two smaller $\alpha$ values the $G E O^{\prime}$ spread within AVDZ is larger than that of AVTZ \& AVQZ, whereas the situation is reversed at the larger two $\alpha$ values. This also suggest that $\alpha$-PBE at $\alpha=0.36$ and with AVDZ provides great geometric performance relative to its cost. 


\subsection{Geometric performance of Grimme's '3c' composite methods}

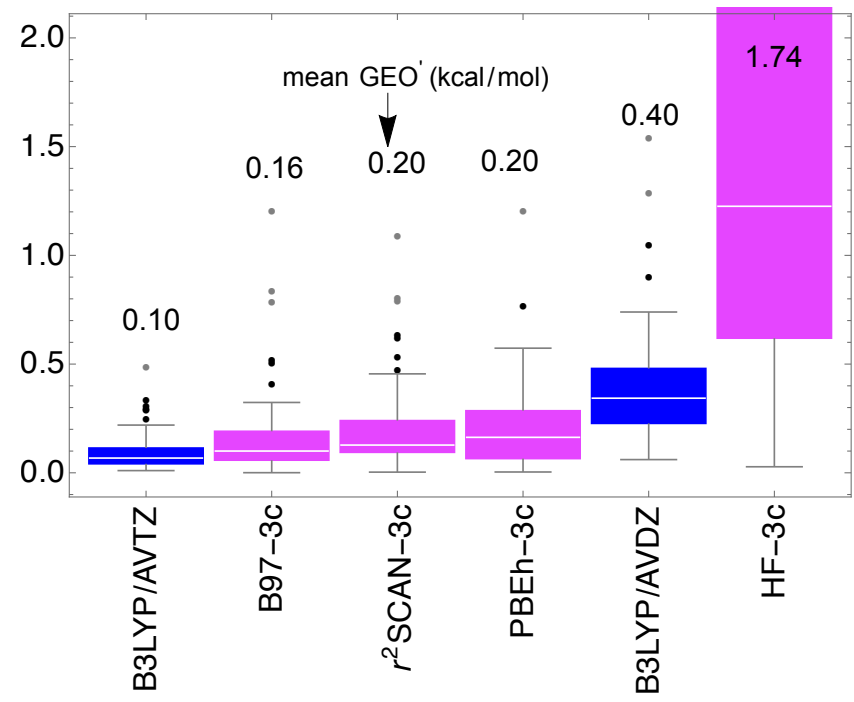

Figure 9: $G E O^{\prime}$ boxplots for the four 3c methods and the B2se dataset. The B3LYP results within AVTZ and AVDZ are shown for comparison. Mean $G E O^{\prime}$ values (kcal/mol) are also reported in the figure. For the same plot, but with a full range in the y-axes, see Figure S11.

In view of their excellent performance to cost ratio, the '-3c' family of composite methods developed by Grimme and co-workers is becoming more and more popular. $\frac{336-38}{38}$ The family includes: HF-3c, $\stackrel{36}{ }$ PBEh-3c, ${ }^{37}$ B97-3c,, 38 and the most recent $r^{2}-\mathrm{SCAN}-3 \mathrm{c}^{3}$ ('a Swiss army knife'), which is built upon the original $r^{2}$-SCAN of Perdew and co-workers. ${ }^{39]}$ These methods are characterized by small, but carefully chosen set of atomic basis functions, and classical potentials designed to correct their electronic structure part. Boxplots with $G E O^{\prime}$ values of the four $3 \mathrm{c}$ methods for $\mathrm{B} 2 \mathrm{se}$ are shown in Figure 9, with B3LYP/AVTZ and B3LYP/AVDZ boxplots shown for comparison. From this figure, we can see that with way larger $G E O^{\prime}$ values, HF-3c stands out from the other three $3 \mathrm{c}$ methods. These three 3c methods have similar mean $G E O^{\prime}$ values, with B97-3c having the lowest mean $G E O^{\prime}$, and $r^{2}$-SCAN-3c having the smallest spread. The three $3 \mathrm{c}$ methods are much better than B3LYP/AVDZ, but get beaten by
B3LYP/AVTZ. Thus, they overall give highly accurate B2se structures relative to their high efficiency.

\section{$4 G E O^{\prime}$ analysis: a break- down of $G E O^{\prime}$ into com- ponents from errors in geometric parameters}

\subsection{GEO' decomposition}

By expanding $E(\mathbf{G})$ around its $\mathbf{G}_{0}$ minimum up to second order, we obtain the harmonic approximation to $G E O:$ [1]

$$
G E O \approx G E O_{\mathrm{h}}=\frac{1}{2} \Delta \tilde{\mathbf{G}}^{\top} \mathbf{H} \Delta \tilde{\mathbf{G}},
$$

where $\mathbf{H}$ is the Hessian of $E(\mathbf{G})$ at the $\mathbf{G}_{0}$ minimum. We can write $G E O^{\prime}$ in the same way, by expanding $\tilde{E}(\mathbf{G})$ around its $\tilde{\mathbf{G}}$ minimum:

$$
G E O^{\prime} \approx G E O_{\mathrm{h}}^{\prime}=\frac{1}{2} \Delta \tilde{\mathbf{G}}^{\top} \tilde{\mathbf{H}} \Delta \tilde{\mathbf{G}},
$$

The difference between $G E O_{\mathrm{h}}^{\prime}$ and $G E O^{\prime}$ is defined here as the measure of the anaharmonicty of $G E O^{\prime}$ :

$$
\Delta_{A}=G E O_{\mathrm{h}}^{\prime}-G E O^{\prime},
$$

which, as we shall see later, is negligibly small for our molecules. Even a further simplification can be obtained if we use $\tilde{\mathbf{H}}_{q}$ in a given set of internal coordinates and neglect its off-diagonal elements. Then $G E O_{\mathrm{h}}^{\prime}$ becomes:

$$
G E O_{\mathrm{h}}^{\prime} \approx G E O_{\mathrm{s}}^{\prime}=\sum_{i}^{3 N-6} \frac{1}{2} \tilde{f}_{i, i}^{q}\left(\Delta q_{i}\right)^{2} .
$$

where $\Delta q_{i}=\tilde{q}_{i}-q$ are the errors in internal coordinates, and $\tilde{f}_{i, i}^{q}$ are the diagonal elements of the $\tilde{\mathbf{H}}_{q}$ Hessian in internal coordinates (force constants). In this way, Eq. 9 enables us to partition $G E O^{\prime}$ into the contributions from the errors in geometric parameters in internal coordinates (bond lengths, bond angles, torsion 
angles, etc.). We also define:

$$
\Delta_{C}=G E O_{\mathrm{s}}^{\prime}-G E O_{\mathrm{h}}^{\prime},
$$

which is a contribution to $G E O^{\prime}$ from the coupling of internal coordinates (due to typically small, but generally non-zero off-diagonal elements of $\tilde{\mathbf{H}}_{q}$ ). Combining Eqs. 8. 10, we can write:

$$
G E O^{\prime}=\Delta_{A}+\Delta_{C}+\frac{1}{2} \sum_{i}^{3 N-6} \tilde{f}_{i, i}^{q}\left(\Delta q_{i}\right)^{2} .
$$

Here, Eqs. 8 11 apply to $G E O^{\prime}$, but one can define analogous equations for $G E O$. As we shall see, the third term on the r.h.s. of Eq. 11 accounts for most of $G E O^{\prime}$, and this enables its intuitive interpretation in terms of the errors in individual geometric parameters. Instead of using internal coordinates to partition $G E O^{\prime}$, one can also use the errors in $G E O^{\prime}$ normal modes (the eigenvectors of $\tilde{\mathbf{H}}$ in e.g., Cartesian coordinates). 1 The advantage of the latter analysis is that $G E O_{\mathrm{s}}^{\prime}$ becomes exactly equal to $G E O^{\prime}$, but the errors in these coordinates are less chemically intuitive than errors in e.g., bond lengths. This is why we proceed with the $G E O^{\prime}$ analysis in internal coordinates.

\subsection{Illustrations}

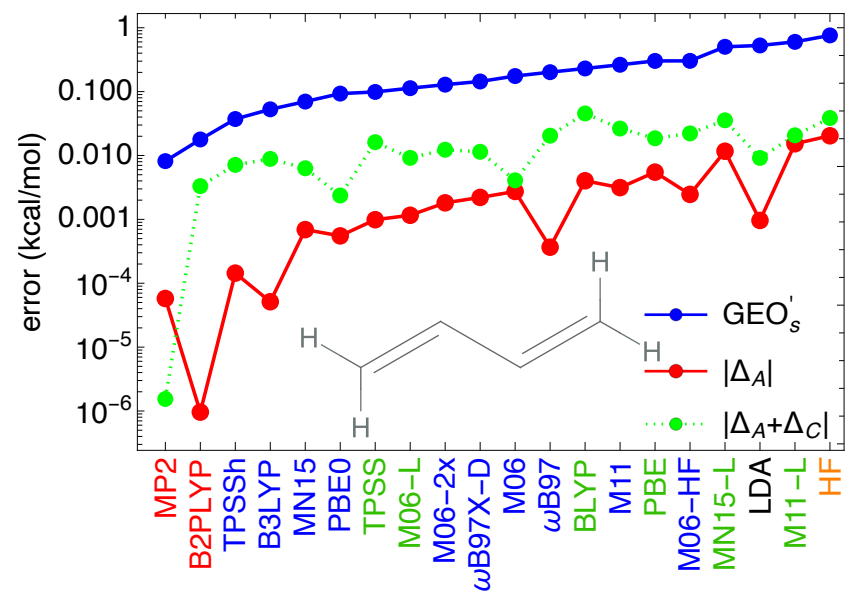

Figure 10: $G E O_{\mathrm{s}}^{\prime}, \Delta_{A}$ and $\Delta_{C}$ values of Eqs. 7 . 10 for the butadiene molecule. Note the logscale in the y-axes. The approximations are ranked by the $G E O_{\mathrm{s}}^{\prime}$ values. The AVQZ basis set is used in tandem with all approximations.
To illustrate the quantities of Eq. 11, we take a butadiene molecule as an example. In Figure 10, we rank the approximations within AVQZ based on their $G E O_{\mathrm{s}}^{\prime}$ values. In the same plot, we also show $\left|\Delta_{A}+\Delta_{C}\right|$ and $\left|\Delta_{A}\right|$ (note the $\log$-scale in the y-axes). From this figure we can see that $\left|\Delta_{A}\right|$ is negligible relative to $G E O_{\mathrm{s}}^{\prime}$, and that $\Delta_{A}+\Delta_{C}$ sum also accounts only for a small fraction of $G E O^{\prime}$. This means that most of $G E O^{\prime}$ can be directly linked and partitioned into the contributions from the errors in specific geometric parameters. This breakdown for the same butadiene molecule is shown in Figure 11 . Based on Eq. 9, we partition $G E O_{\mathrm{s}}^{\prime}$ into contributions from errors in the single and double bond lengths and the remainder of $G E O_{\mathrm{s}}^{\prime}$ here is due to the errors in the angles (the sum of contributions from both bond and torsion angles ). We can also see how these $G E O_{\mathrm{s}}^{\prime}$ components vary as we change the basis sets: AVQZ (left panel), AVTZ (middle panel), and AVDZ (right panel). The contribution from the angles is small for all approximations and basis sets. The $G E O^{\prime}$ weights do not change substantially as we go from AVQZ to AVTZ as it is the case with the total $G E O^{\prime}$ values. Interesting patterns and grouping of functionals can be observed based on their $G E O^{\prime}$ contributions. At the AVQZ and AVTZ level, we can see that the single-bond contribution strongly dominates old-school (semi)local functionals (LDA, BLYP, PBE, TPSS). The situation is different in the case of hybrids, for most of which a double-bond contributions strongly dominates their $G E O^{\prime}$. As we go from AVTZ to AVDZ the relative contributions to $G E O^{\prime}$ change strikingly and so do the rankings of approximations. An immediate noticeable change is that the light blue colour becomes more dominant in the AVDZ bars, indicating a more significant contribution from the errors in single-bond lengths. This change in the basis also reverses the trends in hybrids, where the contribution from singlebonds dominates over that of the double-bonds. On the other hand, a single-bond contribution still dominates $G E O^{\prime}$ of PBE, TPSS and BLYP, with the difference that $G E O^{\prime}$ of these functionals within AVDZ bears a substantial doublebond contribution. As observed earlier, the ge- 


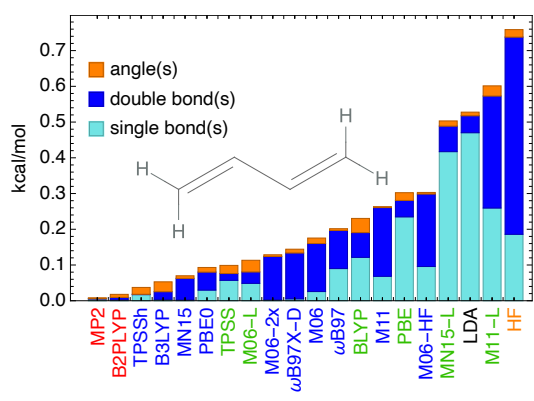

(a) AVQZ

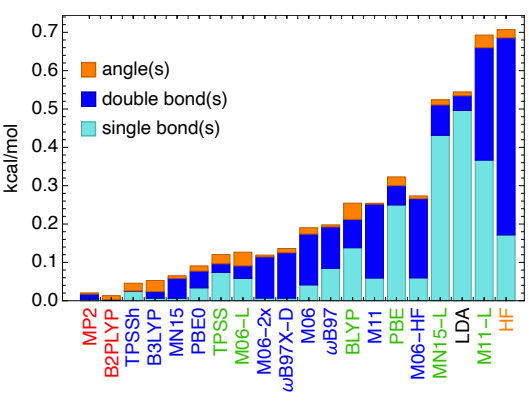

(b) AVTZ

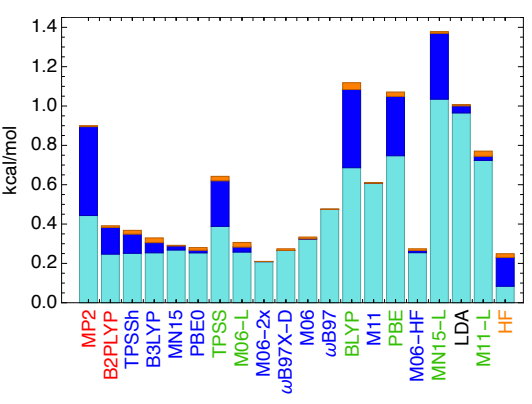

(c) AVDZ

Figure 11: The decomposition of $G E O_{\mathrm{s}}^{\prime}$ for the butadiene molecule into the angle, double bond, and single bond components (Eq. 9). The three panels show the results within different basis sets: AVQZ (left), AVTZ (center), AVDZ (right). The approximations in all three panels are are ranked by their AVQZ GEO values (left panel).

ometric performance of $\mathrm{HF}$ gets drastically better as one goes from AVTZ to AVDZ and we can see here that this change in the basis substantially reduces both single- and double-bond contributions to its $G E O^{\prime}$. At the AVDZ basis, HF gets beaten here only by M06-2X, which also contains a large amount of exact exchange $(54 \%)$.

The same analysis for more molecules is shown in Figure 12. Here the basis set is fixed (AVTZ), but a more detailed analysis with different basis sets and additional molecules is given in Section 56 of the SI. In the case of acetylene molecule [panel (a)] the patterns are even clearer than it was the case with butadiene. Namely, GEO' of LDA, PBE, BLYP and TPSS is almost entirely due to the errors in the triple bond length, whereas $G E O^{\prime}$ of hybrids are almost entirely due to the single bonds. In the case of benzene [panel (b)], the picture is more nuanced: $G E O^{\prime}$ of GGAs, LDA and TPSS is still mostly due the error in the single bond, but has a significant contribution from the errors in the unsaturated $\mathrm{C}-\mathrm{C}$ bond lengths. By looking at the remaining two panels in Figure 12, the similar patterns can be observed: the angle contributions to $G E O_{\mathrm{s}}^{\prime}$ is still small, $G E O_{\mathrm{s}}^{\prime}$ of hybrids is dominated by a single bond component, whereas unsaturated bonds dominate $G E O_{\mathrm{s}}^{\prime}$ of the PBE/BLYP/TPSS group. The -L Minnesota functionals (ones that make no use of exact exchange) behave differently: the $G E O^{\prime}$ weights of MN15-L are similar to those of the PBE/TPSS/BLYP group, whereas the weights of M06-L and M11-L are more similar to those of hybrids. Of course, if the AVDZ basis is used instead, the trends change, as was described by the butadiene example (see Section $\mathbf{S 6}$ for the results for the other basis sets).

\subsection{Breakdown of $G E O^{\prime}$ from $\alpha$-PBE for the formaldehyde molecule}

We could see in Fig 12 that the $G E O^{\prime}$ weights of PBE and PBE0 are substantially different. To shed more light on that and gain insight into the position of the $\alpha$ minimum for $\alpha$-PBE in Figure 7, we consider here how the $\alpha$-PBE $G E O^{\prime}$ components vary for the formaldehyde molecule. This molecule is chosen as its optimal $\alpha$ value is about the same as that for the whole B2se dataset (about 0.2 at the AVTZ basis set). The results are shown in Figure 13. From the top panel, where AVTZ is used, we first note that the $G E O^{\prime}$ curve is accurately described by that of $G E O_{\mathrm{s}}^{\prime}$. Furthermore, the angle contribution to $G E O^{\prime}$ is very small, and thus the $G E O^{\prime}$ black curve is essentially the sum of its single-bonds and double-bond components (the light blue and blue curves). Up to $\alpha \sim 0.15$, most of $G E O^{\prime}$ comes from the single bonds, whereas in the region for $\alpha$ values in between $\sim 0.35$ and $\sim 0.6$, it mostly comes from the double bond. The minimum of the $G E O^{\prime}$ curve (black) is at about $\alpha=0.2$, and is closer to the minimum of the double-bond component 


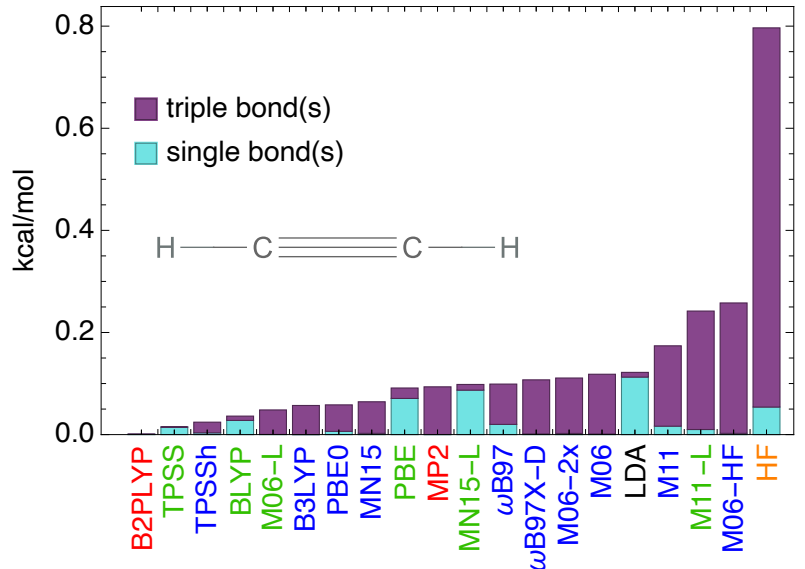

(a)

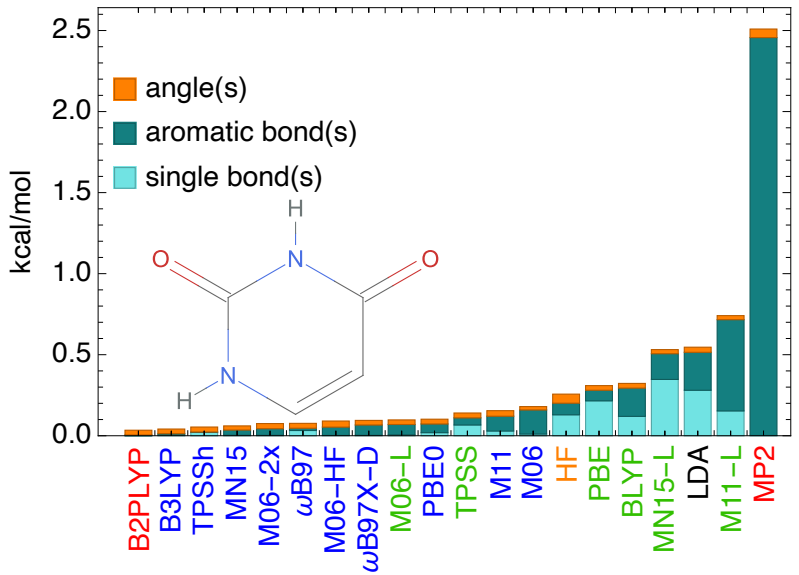

(c)

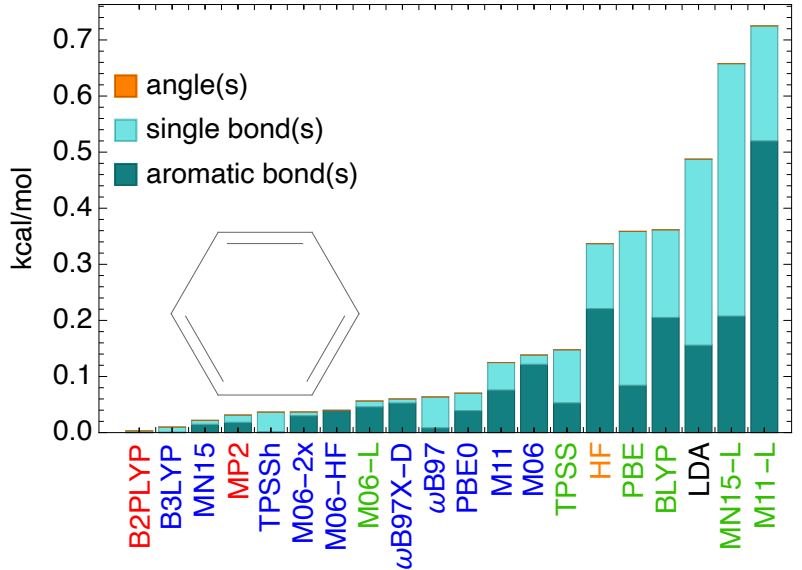

(b)

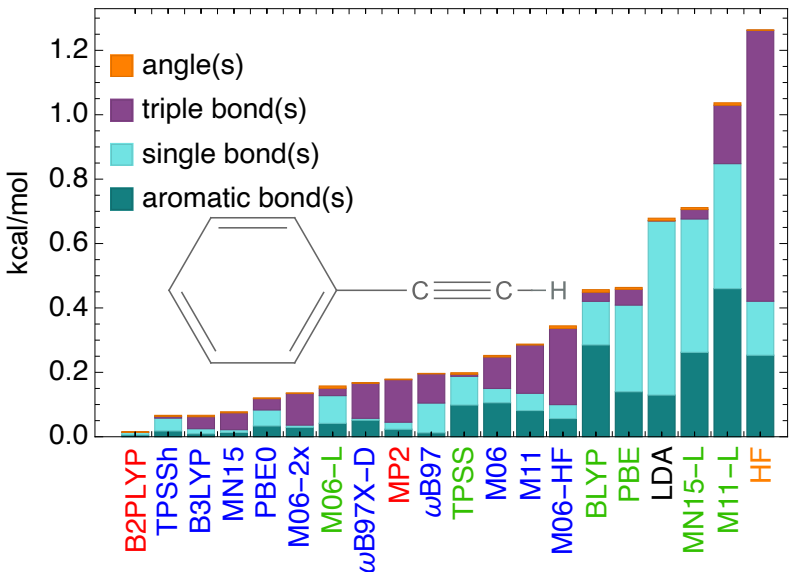

(d)

Figure 12: Different components of $G E O_{\mathrm{s}}^{\prime}$ for a set of approximations and a selection of 6 molecules. The AVTZ basis set is used in all calculations. 'Aromatic bonds' denote the $\mathrm{C}=\mathrm{C}$ bonds inside of the benzene ring. The same analysis for more molecules and basis sets is given in Section S6 of the SI.

( $\alpha \sim 0.09)$ than to that of the single-bonds component $(\alpha \sim 0.45)$, as the latter minimum is shallower. The large distance between the blue and light blue minima do not allow $\alpha$-PBE to be very accurate for both single- and double-bond of formaldehyde at the same time. Thus, at the $\alpha \sim 0.2$ minimum there is some contribution from both bond types. In the bottom panel of Figure 13, we repeat the same plot, but within AVDZ. The situation in this panel is similar to that of the upper panel, with the difference that the position of the minima of the four curves are now shifted towards larger $\alpha$ values (only the angle between the bonds is most accurate at $\alpha=1$ for both basis sets). This observation is in-line with the earlier analysis that focused on the changes in the total $G E O^{\prime}$ values as one goes from AVTZ to AVDZ.

\section{4 $G E O^{\prime}$ analysis for the phenyl radical}

As said earlier, the phenyl radical is the only open-shell species in the B2se set. That is why it was excluded from the $G E O^{\prime}$ statistics given in Section 3, and we analyse it separately here. Figure 14(a) ranks the approximations within AVTZ based on their $G E O^{\prime}$ values for the phenyl radical and compares $G E O^{\prime}$ and $G E O_{\mathrm{s}}^{\prime}$ values. These rankings can be compared to those for the benzene molecule [Figure $12(b)]$. The most obvious difference be- 


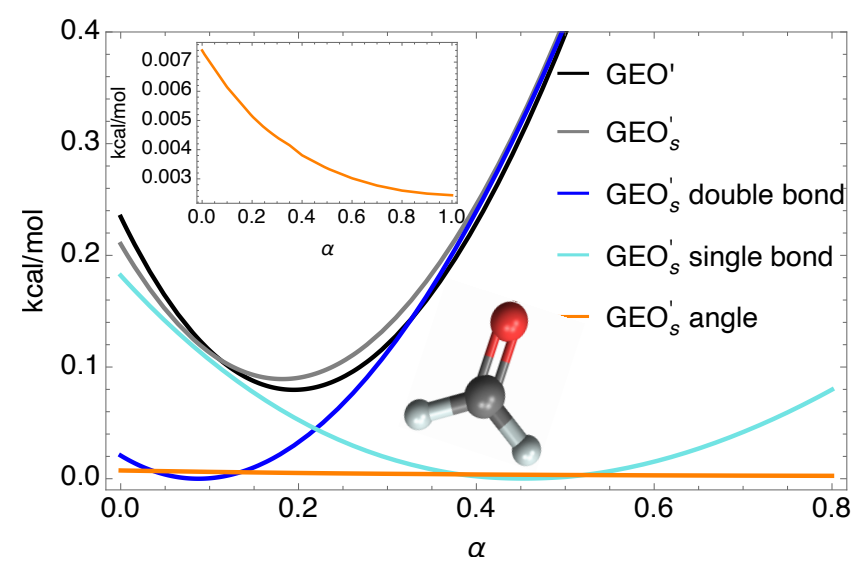

(a) AVTZ

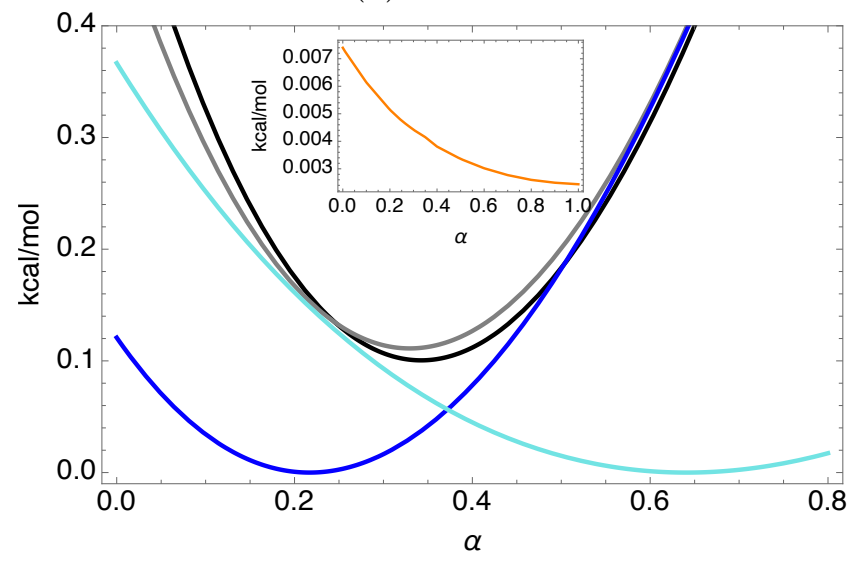

(b) AVDZ

Figure 13: $G E O^{\prime}, G E O_{\mathrm{s}}^{\prime}$ and its angle, singleand double-bond components for $\alpha$-PBE as a function of $\alpha$ for the formaldehyde molecule at the AVTZ (top panel) and AVDZ (bottom panel) basis sets.

tween these two plots is the position of MP2, which was one of the top performers for benzene, whereas it stands out as by far the worst for the phenyl radical. The MP2 calculation here is based on the unrestricted HF (UHF), which is severely spin-contaminated $\left(\left\langle S^{2}\right\rangle\right.$ off by more than $50 \%$ for the considered radical), and in such cases MP2 typically gives very bad geometries. 1140/41 Although spin-contaminated, UHF itself gives way more reasonable geometry for the phenyl radical than MP2 [Figure 14(a)]. The inset of Figure 14(a) explores $G E O^{\prime}$ values for different methods built upon MP2. It is of no surprise ${ }^{42}$ that $G E O^{\prime}$ of SCS-MP2 ${ }^{40}$ is just slightly lower than that of MP2, as SCS-MP2 also uses the UHF orbitals here. On the other hand, B2PLYP and the two orbital-optimized (OO) MP2 approaches (OOMP2 and OO-SCS-MP2 $23-45)$ reduce spin contamination of MP2, and thus give highly accurate structures for the phenyl radical $\left(G E O^{\prime}\right.$ values $\sim 0.05 \mathrm{kcal} / \mathrm{mol}$ ). One should also note that B2PLYP is the cheapest of the three methods as its cost is about the same as MP2, while orbital optimizations make the two OO-MP2 approaches substantially more expensive than MP2.

In Figure 14(b), we decompose $G E O_{\mathrm{s}}^{\prime}$ into the contributions from $\mathrm{C}-\mathrm{H}$ and $\mathrm{C}=\mathrm{C}$ bonds, and angles. These results for the phenyl radical can also be compared with those for the benzene molecule [Figure 12(b)]. From this plot, we can see that large $G E O^{\prime}$ of MP2 comes almost exclusively from the errors in the $\mathrm{C}=\mathrm{C}$ bond lengths. Instead of summing contributions for the same bond types, we can also measure the $G E O^{\prime}$ contributions from each bond. While a more detailed analysis is shown in the SI (Fig. S34), we focus on MP2 in Figure 12(c), where for each unique bond length we show errors in pm. We can also see how each of these translate to $G E O^{\prime}$ contributions, which are obtained by squaring the error and multiplying it by half of the underlying force constant (Eq. 9). We can see that the MP2 errors in $\mathrm{C}=-\mathrm{C}$ bond lengths are very large and range from $\sim 2.5 \mathrm{pm}$ to $\sim 3 \mathrm{pm}$ resulting in $G E O^{\prime}$ contributions from 0.3 to $0.5 \mathrm{kcal} / \mathrm{mol}$ per bond. The errors in the $\mathrm{C}-\mathrm{H}$ bond lengths are much smaller, and result in way smaller $G E O_{\mathrm{s}}^{\prime}$ contributions given that: $G E O^{\prime}$ grows quadratically with the errors in geometric parameters and given the smaller value of the $f_{C-H}$ than $f_{\mathrm{C}=-\mathrm{C}}$ force constant. In contrast to the large MP2 errors in $\mathrm{C}=\mathrm{C}$ bonds, those of B2PLYP are much smaller (within 0.16 pm resulting in negligible $G E O^{\prime}$ contributions, $\sim 0.001 \mathrm{kcal} / \mathrm{mol}$ ). 


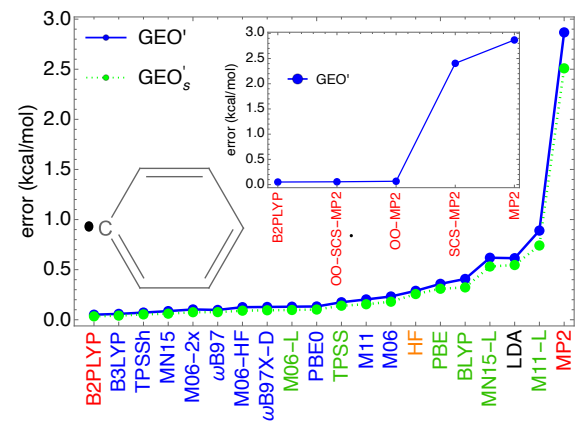

(a)

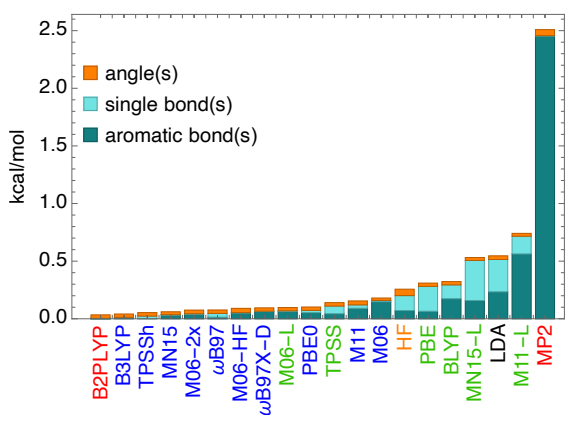

(b)

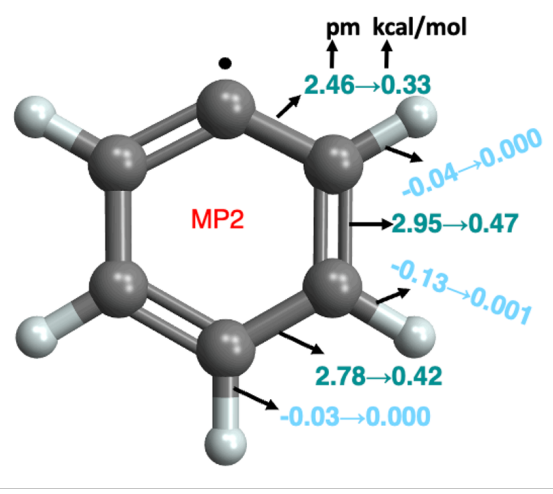

(c)

Figure 14: $G E O^{\prime}$ analysis for the phenyl radical. Panel (a) compares $G E O^{\prime}$ and $G E O_{\mathrm{s}}^{\prime}$ for a range of approximate methods. Panel (b) shows a breakdown of $G E O_{\mathrm{s}}^{\prime}$ into components due to the errors in: angles, $\mathrm{C}-\mathrm{H}$ bond lengths ('single bonds'), and $\mathrm{C}=\mathrm{C}$ bonds ('aromatic bonds'). Panel (c) shows errors in bond lengths in pm of MP2 and how each of them translate to $G E O_{\mathrm{s}}^{\prime}$ contribution in $\mathrm{kcal} / \mathrm{mol}$ (Eq. 9). AVTZ basis set is used in all calculations.

\section{Conclusion and outlook}

The present paper demonstrates the usability of $G E O^{\prime}$ for quantifying and analysing geometric errors in approximate molecular structures. The use of $G E O^{\prime}$ in place of $G E O$ in tandem with semiexperimental geometries greatly simplifies the whole analysis and enables us to bypass a need for using the input from expensive correlated wavefunction calculations, e.g., $\operatorname{CCSD}(\mathrm{T})$. With the $G E O^{\prime}$ analysis, we identify patterns in geometric performance across different classes of approximations and basis sets. The focus here is on main-group structures, but the developed tools are widely applicable and can be used in a straightforward way to quantify and analyse geometric errors for any molecule and any approximation in simple and chemically intuitive terms.

Both $G E O$ and $G E O^{\prime}$ use energy units to assess qualities of approximate geometries (unlike other measures for geometric errors, such as averages in errors in geometric parameters). Several advantages arise from that. First, $G E O^{\prime}$ and $G E O$ can be directly compared to existing energetic scores of electronic structure methods or can be included in new ones. For this reason, we recommend the inclusion of mean $G E O^{\prime}$ values for the B2se set to new versions of energetic scores, such as WTMAD-2 scores pertaining to the GMTKN55 collection of databases. $\stackrel{46 / 47}{ }$ Sec- ond, mean $G E O^{\prime}$ for B2se can also be included in the training of new empirical methods, since the resulting methods would likely have better geometric performance than those using the same form but trained only on standard energetic datasets (e.g., datasets with atomization energies, barrier heights, binding energies, etc.) $6 \sqrt{48}$

Here the focus is on ground-state structures, but in the future work GEO will also be calculated for excited-state structures providing tests for TD-DFT and wave-function methods. $26 \mid 49$ The same or slightly adjusted analysis will also be applied to noncovalent structures, ${ }^{5 / 50}$ transition states (enabling quantification of geometric errors for barrier heights), as well as the structures of large transition-metal complexes obtained from semiempirical methods, for which DFT structures should be sufficiently good reference. ${ }^{51}$ In the context of DFT, improved densities should yield improved geometries. ${ }^{52}$ Thus, in addition to the standard DFT, we will also test the geometric performance of its densitycorrected variant. $48[53,55$

\section{Associated content}

The Supporting Information is available free of charge at..., and it contains computational details and additional results references through- 
out the main text (PDF). Data for $G E O^{\prime}$ values of approximations for the B2se molecules are also provided (xlsx).

Acknowledgement This project has received funding from the European Union's Horizon 2020 research and innovation programme under the Marie Skłodowska-Curie grant agreement 101033630, and in part by NSF (CHEM 1856165). Computational resources for the present project have been provided through NWO's Vici grant 724.017.001. I also thank Dr. Peter Kraus for suggesting the use of semiexperimental geometries in the context of the present work.

\section{A Further details on $G E O$ and $G E O^{\prime}$}

For an approximate electronic structure method, the total error is given by:

$$
\Delta E=\tilde{E}(\tilde{\mathbf{G}})-E\left(\mathbf{G}_{0}\right),
$$

and thus contains errors both due to the approximate geometry and approximate energy (see Section 1 for the definition of the quantities in Eq 12). To decompose this error into GEO and non-geometric part ('purely energetic components', denoted by $P$ and $P^{\prime}$ below), we add and subtract $E(\tilde{\mathbf{G}})$ to the r.h.s of Eq. 12 .

$$
\Delta E=\underbrace{E(\tilde{\mathbf{G}})-E\left(\mathbf{G}_{0}\right)}_{G E O \geq 0}+\underbrace{\tilde{E}(\tilde{\mathbf{G}})-E(\tilde{\mathbf{G}})}_{P} .
$$

Adding and subtracting $\tilde{E}\left(\mathbf{G}_{0}\right)$ to the r.h.s of Eq. 12 we obtain an alternative form of Eq. 13 .

$$
\Delta E=\underbrace{\tilde{E}(\tilde{\mathbf{G}})-\tilde{E}\left(\mathbf{G}_{0}\right)}_{-G E O^{\prime} \leq 0}+\underbrace{\tilde{E}\left(\mathbf{G}_{0}\right)-E\left(\mathbf{G}_{0}\right)}_{P^{\prime}} .
$$

The signs of $G E O$ and $G E O^{\prime}$ also dictate the following chain of inequalities:

$$
P \leq \Delta E \leq P^{\prime}
$$

Since $G E O$ is typically very accurately approx- imated by $G E O^{\prime}$, then we also have: $G E O \approx$ $\frac{1}{2}\left(P^{\prime}-P\right)$. As discussed in Ref. 1, for equilibrium structures (minima of potential energy surfaces), $G E O$ and $G E O^{\prime}$ are always positive, whereas their signs for transition states (the first order saddle points of potential energy surfaces) are not definite.

\section{References}

(1) Vuckovic, S.; Burke, K. Quantifying and Understanding Errors in Molecular Geometries. The Journal of Physical Chemistry Letters 2020, 11, 9957-9964.

(2) Su, N. Q.; Xu, X. Beyond energies: Geometry predictions with the XYG3 type of doubly hybrid density functionals. Chemical Communications 2016, 52, 1384013860 .

(3) Grimme, S.; Hansen, A.; Ehlert, S.; Mewes, J.-M. r2SCAN-3c: A "Swiss army knife" composite electronic-structure method. The Journal of Chemical Physics 2021, 154, 064103.

(4) Helgaker, T.; Gauss, J.; Jo/rgensen, P.; Olsen, J. The prediction of molecular equilibrium structures by the standard electronic wave functions. The Journal of Chemical Physics 1997, 106, 6430-6440.

(5) Witte, J.; Goldey, M.; Neaton, J. B.; Head-Gordon, M. Beyond Energies: Geometries of Nonbonded Molecular Complexes as Metrics for Assessing Electronic Structure Approaches. 2015, 11, 14811492.

(6) Mardirossian, N.; Head-Gordon, M. Thirty years of density functional theory in computational chemistry: an overview and extensive assessment of 200 density functionals. Mol. Phys. 2017, 115, 2315-2372.

(7) Najibi, A.; Goerigk, L. DFT-D4 counterparts of leading meta-generalized-gradient approximation and hybrid density functionals for energetics and geometries. 
Journal of Computational Chemistry 2020, 41, 2562-2572.

(8) Verma, P.; Truhlar, D. G. Status and challenges of density functional theory. Trends in Chemistry 2020, 2, 302-318.

(9) Bannwarth, C.; Caldeweyher, E.; Ehlert, S.; Hansen, A.; Pracht, P.; Seibert, J.; Spicher, S.; Grimme, S. Extended tight-binding quantum chemistry methods. Wiley Interdisciplinary Reviews: Computational Molecular Science 2021, 11, e1493.

(10) Piccardo, M.; Penocchio, E.; Puzzarini, C.; Biczysko, M.; Barone, V. Semiexperimental equilibrium structure determinations by employing B3LYP/SNSD anharmonic force fields: Validation and application to semirigid organic molecules. The Journal of Physical Chemistry A 2015, 119, 2058-2082.

(11) Spackman, P. R.; Jayatilaka, D.; Karton, A. Basis set convergence of CCSD (T) equilibrium geometries using a large and diverse set of molecular structures. The Journal of chemical physics 2016, 145, 104101.

(12) Dunning, T. H.; Peterson, K. A.; Wilson, A. K. Gaussian basis sets for use in correlated molecular calculations. X. The atoms aluminum through argon revisited. The Journal of Chemical Physics 2001, 114, 9244-9253.

(13) Grimme, S. Semiempirical hybrid density functional with perturbative secondorder correlation. The Journal of chemical physics 2006, 124, 034108.

(14) Bakowies, D.; von Lilienfeld, O. A. Density Functional Geometries and ZeroPoint Energies in Ab Initio Thermochemical Treatments of Compounds with FirstRow Atoms (H, C, N, O, F). Journal of Chemical Theory and Computation 2021, 17, 4872-4890.
(15) Penocchio, E.; Piccardo, M.; Barone, V. Semiexperimental Equilibrium Structures for Building Blocks of Organic and Biological Molecules: The B2PLYP Route. Journal of Chemical Theory and Computation 2015, 11, 4689-4707.

(16) Puzzarini, C.; Barone, V. Diving for Accurate Structures in the Ocean of Molecular Systems with the Help of Spectroscopy and Quantum Chemistry. Accounts of Chemical Research 2018, 51, 548-556.

(17) Pulay, P.; Meyer, W.; Boggs, J. E. Cubic force constants and equilibrium geometry of methane from Hartree-Fock and correlated wavefunctions. The Journal of Chemical Physics 1978, 68, 5077-5085.

(18) Woon, D. E.; Dunning Jr, T. H. Gaussian basis sets for use in correlated molecular calculations. V. Core-valence basis sets for boron through neon. J. Chem. Phys. 1995, 103, 4572-4585.

(19) Zhao, Y.; Truhlar, D. G. A new local density functional for main-group thermochemistry, transition metal bonding, thermochemical kinetics, and noncovalent interactions. The Journal of Chemical Physics 2006, 125, 194101.

(20) Zhao, Y.; Truhlar, D. G. Density Functional for Spectroscopy: No Long-Range Self-Interaction Error, Good Performance for Rydberg and Charge-Transfer States, and Better Performance on Average than B3LYP for Ground States. The Journal of Physical Chemistry A 2006, 110, 1312613130.

(21) Perdew, J. P.; Burke, K.; Ernzerhof, M. Generalized Gradient Approximation Made Simple. Phys. Rev. Lett. 1996, $77,3865$.

(22) Becke, A. D. Density-functional exchangeenergy approximation with correct asymptotic behavior. Phys. Rev. A 1988, 38, 3098 . 
(23) Lee, C.; Yang, W.; Parr, R. G. Development of the Colle-Salvetti correlationenergy formula into a functional of the electron density. Physical review B 1988, 37, 785 .

(24) Goerigk, L.; Grimme, S. A thorough benchmark of density functional methods for general main group thermochemistry, kinetics, and noncovalent interactions. Physical Chemistry Chemical Physics 2011, 13, 6670-6688.

(25) Peverati, R.; Truhlar, D. G. M11-L: A Local Density Functional That Provides Improved Accuracy for Electronic Structure Calculations in Chemistry and Physics. The Journal of Physical Chemistry Letters 2011, 3, 117-124.

(26) Bremond, E.; Savarese, M.; Adamo, C.; Jacquemin, D. Accuracy of TD-DFT geometries: a fresh look. Journal of chemical theory and computation 2018, 14, 37153727 .

(27) Zhao, Y.; Truhlar, D. G. The M06 suite of density functionals for main group thermochemistry, thermochemical kinetics, noncovalent interactions, excited states, and transition elements: two new functionals and systematic testing of four M06-class functionals and 12 other functionals. Theoretical Chemistry Accounts 2007, 120, 215-241.

(28) Yu, H. S.; He, X.; Truhlar, D. G. MN15-L: A New Local Exchange-Correlation Functional for Kohn-Sham Density Functional Theory with Broad Accuracy for Atoms, Molecules, and Solids. Journal of Chemical Theory and Computation 2016, 12, 1280-1293.

(29) Grimme, S.; Antony, J.; Ehrlich, S.; Krieg, H. A consistent and accurate ab initio parametrization of density functional dispersion correction (DFT-D) for the 94 elements $\mathrm{H}-\mathrm{Pu}$. The Journal of chemical physics 2010, 132, 154104.
(30) Becke, A. D.; Johnson, E. R. A densityfunctional model of the dispersion interaction. The Journal of chemical physics 2005, 123, 154101.

(31) Grimme, S.; Ehrlich, S.; Goerigk, L. Effect of the damping function in dispersion corrected density functional theory. Journal of computational chemistry 2011, 32, $1456-1465$.

(32) Staroverov, V. N.; Scuseria, G. E.; Tao, J.; Perdew, J. P. Comparative assessment of a new nonempirical density functional: Molecules and hydrogen-bonded complexes. The Journal of chemical physics 2003, 119, 12129-12137.

(33) Goerigk, L.; Reimers, J. R. Efficient Methods for the Quantum Chemical Treatment of Protein Structures: The Effects of London-Dispersion and Basis-Set Incompleteness on Peptide and Water-Cluster Geometries. Journal of Chemical Theory and Computation 2013, 9, 3240-3251.

(34) Perdew, J. P.; Ernzerhof, M.; Burke, K. Rationale for mixing exact exchange with density functional approximations. $J$. Chem. Phys. 1996, 105, 9982-9985.

(35) Adamo, C.; Barone, V. Toward reliable density functional methods without adjustable parameters: The PBE0 model. The Journal of chemical physics 1999, 110, 6158-6170.

(36) Sure, R.; Grimme, S. Corrected small basis set Hartree-Fock method for large systems. Journal of computational chemistry 2013, 34, 1672-1685.

(37) Grimme, S.; Brandenburg, J. G.; Bannwarth, C.; Hansen, A. Consistent structures and interactions by density functional theory with small atomic orbital basis sets. The Journal of chemical physics 2015, 143, 054107.

(38) Brandenburg, J. G.; Bannwarth, C.; Hansen, A.; Grimme, S. B97-3c: A revised low-cost variant of the B97-D den- 
sity functional method. The Journal of Chemical Physics 2018, $148,064104$.

(39) Furness, J. W.; Kaplan, A. D.; Ning, J.; Perdew, J. P.; Sun, J. Accurate and Numerically Efficient r2SCAN MetaGeneralized Gradient Approximation. The Journal of Physical Chemistry Letters 2020, 11, 8208-8215.

(40) Grimme, S. Improved second-order Møller-Plesset perturbation theory by separate scaling of parallel- and antiparallel-spin pair correlation energies. The Journal of Chemical Physics 2003, 118, 9095-9102.

(41) Shirazi, R. G.; Pantazis, D. A.; Neese, F. Performance of density functional theory and orbital-optimised second-order perturbation theory methods for geometries and singlet-triplet state splittings of arylcarbenes. Molecular Physics 2020, 118, e1764644.

(42) Shirazi, R. G.; Neese, F.; Pantazis, D. A. Accurate Spin-State Energetics for Aryl Carbenes. Journal of Chemical Theory and Computation 2018, 14, 4733-4746.

(43) Lochan, R. C.; Head-Gordon, M. Orbitaloptimized opposite-spin scaled secondorder correlation: An economical method to improve the description of openshell molecules. The Journal of chemical physics 2007, 126, 164101.

(44) Neese, F.; Schwabe, T.; Kossmann, S.; Schirmer, B.; Grimme, S. Assessment of orbital-optimized, spin-component scaled second-order many-body perturbation theory for thermochemistry and kinetics. Journal of chemical theory and computation 2009, 5, 3060-3073.

(45) Kossmann, S.; Neese, F. Correlated ab initio spin densities for larger molecules: orbital-optimized spin-component-scaled MP2 method. The Journal of Physical Chemistry A 2010, 114, 11768-11781.
(46) Goerigk, L.; Hansen, A.; Bauer, C.; Ehrlich, S.; Najibi, A.; Grimme, S. A look at the density functional theory zoo with the advanced GMTKN55 database for general main group thermochemistry, kinetics and noncovalent interactions. Physical Chemistry Chemical Physics 2017, 19, 32184-32215.

(47) Gould, T. 'Diet GMTKN55' offers accelerated benchmarking through a representative subset approach. Physical Chemistry Chemical Physics 2018, 20, 27735-27739.

(48) Song, S.; Vuckovic, S.; Sim, E.; Burke, K. Density sensitivity of empirical functionals. The journal of physical chemistry letters 2021, 12, 800-807.

(49) Loos, P.-F.; Jacquemin, D. Chemically Accurate 0-0 Energies with Not-soAccurate Excited State Geometries. 2019, 15, 2481-2491.

(50) Kraus, P.; Frank, I. Density Functional Theory for Microwave Spectroscopy of Noncovalent Complexes: A Benchmark Study. The Journal of Physical Chemistry A 2018, 122, 4894-4901.

(51) Bursch, M.; Neugebauer, H.; Grimme, S. Structure Optimisation of Large Transition-Metal Complexes with Extended Tight-Binding Methods. 2019, 58, 11078-11087.

(52) Kim, M.-C.; Sim, E.; Burke, K. Ions in solution: Density corrected density functional theory (DC-DFT). J. Chem. Phys. 2014, 140, 18A528.

(53) Kim, M.-C.; Sim, E.; Burke, K. Understanding and reducing errors in density functional calculations. Phys. Rev. Lett. 2013, 111, 073003.

(54) Vuckovic, S.; Song, S.; Kozlowski, J.; Sim, E.; Burke, K. Density functional analysis: The theory of density-corrected DFT. Journal of chemical theory and computation 2019, 
(55) Song, S.; Vuckovic, S.; Sim, E.; Burke, K. Density-corrected DFT explained: Questions and answers. arXiv preprint arXiv:2110.07849 2021, 